\title{
miRNA-200b Signature in the Prevention of Skin Cancer Stem Cells by Polyphenol-enriched Blueberry Preparation
}

\author{
Nawal Alsadi', Jean-François Mallet', Chantal Matar, ${ }^{1,2}$ \\ ${ }^{1}$ Department of Cellular and Molecular Medicine, Faculty of Medicine, University of Ottawa, ${ }^{2}$ Department of Nutrition \\ Sciences, Faculty of Health Sciences, University of Ottawa, Ottawa, Canada
}

\begin{abstract}
Exposure of the skin to solar UV radiation leads to inflammation, DNA damage, and dysregulation of cellular signaling pathways, which may cause skin cancer. Photochemoprevention with natural products is an effective strategy for the control of cutaneous neoplasia. Polyphenols have been proven to help prevent skin cancer and to inhibit the growth of cancer stem cells (CSCs) through epigenetic mechanisms, including modulation of microRNAs expression. Thus, the current study aimed to assess the effect of polyphenol enriched blueberry preparation (PEBP) or non-fermented blueberry juice (NBJ) on expression of miRNAs and target proteins associated with different clinicopathological characteristics of skin cancer such as stemness, motility, and invasiveness. We observed that PEBP significantly inhibited the proliferation of skin CSCs derived from different melanoma cell lines, HS 294T and B16F10. Moreover, PEBP was able to reduce the formation of melanophores. We also showed that the expression of the CD133 stem cell marker in B16F10 and HS294T cell lines was significantly decreased after treating the cells with PEBP in comparison to the NBJ and control groups. Importantly, tumor suppressors' miR-200s, involved in the regulation of the epithelial-to-mesenchymal transition and metastasis, were strikingly upregulated. In addition, we have shown that a protein target of the tumor suppressor miR200b, ZEB1, was also significantly modulated. Thus, the results demonstrates that PEBP possesses potent anticancer and anti-metastatic potentials and may represent a novel chemopreventative agent against skin cancer.
\end{abstract}

Key Words Polyphenols, Cancer stem cells, Neoplasm metastasis, MicroRNA-200b, Zinc finger E-box binding homeobox 1

\section{INTRODUCTION}

Epidemiological studies suggest that the consumption of foods containing antioxidants and polyphenolic compounds can reduce the risk for several types of cancer [1]. Polyphenols from fruits, vegetables, grape seeds, and green tea have been shown to protect the skin from the adverse effects of solar UV radiation [2]. Skin cancer is rapidly increasing and is considered a common malignancy in Canada and the United States [3]. Blueberries represent a well-known source of natural antioxidants, polyphenols, and anthocyanins, with proven anti-proliferative and apoptotic effects on cancer cells [4]. We have previously reported that polyphenol enriched blueberry preparation (PEBP) is significantly involved in cancer chemoprevention and chemotherapy [5]. The fermentation process during PEBP's preparation mediated by a novel bacterium, Serratia vaccini isolated from the blueberry flora, increased its polyphenolic content and endowed it with anti-inflammatory
[6] and antidiabetic properties [4].

The fermentation process increases the bioefficacy and the bioavailability by affecting the structure of the polyphenol compared to its non-fermented counterpart [7]. Moreover, this process also affect the molecular mechanism which is related to the anti-inflammatory activity of plyphenols by decreasing inflammatory signals in pathways mediated by STAT3, phosphatidylinositol 3-kinase (PI3K), extracellular signal kinase $1 / 2$ (ERK1/2), etc. and also controlling the growth of cancer stem cell (CSC) in mammary carcinomas in vitro, in ex vivo and in in vivo settings [5].

CSCs are a small subset of neoplastic cells in solid tumors that give rise to differentiated cancer progeny $[8,9]$. Differentiated cells constitute the bulk of the tumor but are not responsible for metastasis. Thus, it is predicted that CSCs are responsible for tumor growth, maintenance and recurrence [10]. CSCs have many functional characteristics, including the potential to differentiate into several cell types and the ca-

Received June 24, 2021, Revised September 18, 2021, Accepted September 23, 2021

Correspondence to Chantal Matar, E-mail: Chantal.matar@uottawa.ca, https://orcid.org/0000-0003-0346-4751

Check for updates 
pacity for self-renewal [11]. In melanoma skin cancer, CSCs have the ability to grow as spheres, which are collections of cells that arise from a single cell through clonal growth [12], and can be identified by the expression of some markers like CD133 (AC133) [13]. Some of distinct CSCs may undergo the epithelial-to-mesenchymal transition (EMT), which is associated with metastasis [14]. The EMT permits cells to migrate and invade nearby tissues and enter the blood stream while retaining stem cell characteristics, leading to metastasis [14]. Further, CSCs have been found to secrete many growth factors to stimulate tumor growth and appear to confer intrinsic resistance to chemotherapy [15].

EMT is mainly regulated by the transcription factor zinc-finger and E-box binding homeobox (ZEB1/2). ZEB1 can serve as a transcriptional activator and a direct target of mesenchymal genes [16]. ZEB1 is associated with the activation of mesenchymal genes and repression of epithelial genes [17]. The expression of ZEB1 can be inhibited by E-cadherin which plays a critical role in cell to cell adhesion and the metastasis of a variety of tumors [18]. Interestingly, activation of E-cadherin was accompanied by the inhibition of both ZEB1/2 which appeared synergistic [19]. Thus, the overexpression of ZEB1/2 can prevent invasion and metastasis in several cancers by influencing the EMT [17].

Epigenetic mechanisms appear to play a fundamental role in CSC biology, particularly by expressing microRNAs (miRNAs) [20]. miRNAs represent a subset of endogenous small noncoding RNA molecules [21]. Their main function is to down-regulate gene expression in different ways, such as inhibiting mRNA translation or promoting mRNA degradation [22]. miRNAs are overexpressed or down-regulated in malignant tissues, and some can function as both tumor suppressors and oncogenes [23]. In particular, miR-200 family is highly associated with the regulation of CSCs in several cancer types and is involved in one of the most critical steps of the metastatic cascade, the EMT [24,25]. miR-200b is commonly down-regulated in primary melanomas compared with benign nevi [26]. Alternatively, its increased expression has been shown to suppress tumor invasion, proliferation, and metastasis [27]. The expression of ZEB proteins is controlled by miR-200b. For instance, miR-200b suppresses metastasis and migration by down regulating ZEB1 and inducing E-cadherin expression [24].

Given the significant role of epigenetic regulation in CSCs formation and our previous report on the repression of breast CSCs by PEBP [5], the current study aimed to investigate the possible mechanism of action of PEBP against development of skin cancer through regulation of miRNA expression signature and target inflammatory/oncogenic factors involved in survival/stemness pathways in skin CSCs.

\section{MATERIALS AND METHODS}

\section{Preparation of blueberry mixture}

Fully matured wild blueberries (Vaccinium angustifolium Ait.) were harvested from selected areas of the Atlantic region as fresh and untreated fruits. Blueberries were then centrifuged at $500 \times g$ for 10 minutes in an IEC Centra MP4R centrifuge (International Equipment Company, Needham Heights, MA, USA) in order to remove fruit skin and non-homogenized particles. Finally, the juice was sterilized by filtration through a $0.22 \mu \mathrm{m}$ Express Millipore filter apparatus (Millipore, Etobicoke, ON, Canada).

\section{Phenolic compounds}

The Folin-Ciocalteu assay (Across International, Livingston, NJ, USA) was used to measure the total phenolic and polyphenol antioxidants present in the blueberry mixture in a 96 well plates. Extracts were acclimated to room temperature before being analyzed. Gallic acid was used to establish the standard curve. To determine the total phenolic content, Folin-Ciocalteu reagent was diluted with de-ionized water in ratio of 1:2. Two hundred $\mu \mathrm{L}$ of Folin-Ciocalteu reagent was added and neutralized with $25 \mu \mathrm{L}$ of $75 \mathrm{mg} / \mathrm{mL}$ saturated sodium carbonate. After gentle shaking, the mixtures were incubated at room temperature in a dark room for 1 hour. The prepared samples were measured at a wavelength of $700 \mathrm{~nm}$ in a $\mu$-Quant microplate reader (Bio-Tek, Winooski, VT, USA). Absorbance values of the PEBP were calculated based on the standard curve constructed from the gallic acid standards. Thus, the effective concentration of PEBP was expressed as gallic acid equivalents (GAE).

\section{Cell culture}

The HS 294T (ATCC HTB-140) human malignant melanoma cells and B16F10 (ATCC CRL-6475) murine malignant melanoma cells were obtained from the American Type Cell Collection (ATCC; Chicago, IL, USA). The cells were maintained in Dulbecco's Modified Eagle's Medium (DMEM) (no. 11995065; Gibco, Grand Island, NY, USA) supplemented with $10 \%$ (v/v) FBS (no. 30-2020; Gibco) and $0.05 \mathrm{mg} / \mathrm{mL}$ of penicillin/streptomycin (J160007; Sigma-Aldrich, Oakville, ON, Canada) at $37^{\circ} \mathrm{C}$ in a humidified atmosphere with $5 \% \mathrm{CO}_{2}$.

\section{Spheroid formation assay}

Adherent cells were detached by trypsin and single cells were counted using the Countess automated cell counter (Invitrogen, Burlington, ON, Canada). Single cells were plated on Costar ultra-low attachment plates (no. 07200601; Corning, Saint-Laurent, QC, Canada) at $10^{5}$ cells $/ 0.2 \mathrm{~mL} /$ well, in the presence or absence of PEBP and NBJ (non-fermented blueberry juice), in DMEM-F12 (no.12660; Invitrogen), supplemented with $20 \mu \mathrm{g} / \mathrm{mL}$ EGF (no. E9644; Sigma Aldrich), $20 \mu \mathrm{g} / \mathrm{mL}$ basic human fibroblast growth factor (no. F0291; Sigma Aldrich), 10 mg/mL Insulin solution (no. 19278; Sigma 
Aldrich), 100 mM sodium pyruvate (S8636; Sigma-Aldrich), 250 mM L-glutamine (no. G6392; Sigma Aldrich), $100 \mu \mathrm{g} /$ mL hydrocortisone (no. H0135; Sigma Aldrich), and penicillin/ streptomycin (1,000×) (J160007; Sigma-Aldrich). Cells are grown in these conditions as non-adherent spherical clusters, and spheroids were counted after 2 to 3 days by light microscopy.

\section{Flow cytometry}

Cells were dissociated after 24 hours, washed twice with icecold PBS (Sigma-Aldrich), and resuspended. Combinations of monoclonal antibodies against mice were added to the cells, followed by incubation in the dark for 30 minutes at $4^{\circ} \mathrm{C}$. The antibodies against CD24-APC (no. 17-0242-82; BD Bioscience, San Jose, CA, USA), CD44-PE/Cy7 (no. 25-044181; BD Bioscience), and CD133-FITC (no. 11-1331-82; BD Bioscience) were used for B16F10 cells. Antibodies against CD135-APC (no. 130-098-829; BD Bioscience) and CD20VF450 (no. 92590; BD Bioscience) were used for HS294T cells. Labeled spheroid cells were washed twice with PBS to eliminate unbound antibody. Flow cytometry was performed using a Beckman Coulter MoFlo ${ }^{\mathrm{TM}}$ XDP (BD Biosciences Pharmingen, San Francisco, CA, USA). 4',6-Diamidino-2-phenylindole was used to separate dead/dying cells from healthy cells. Side-scatter and forward-scatter profiles were used to eliminate cell doublets.

\section{Cell motility assay}

Cells were plated overnight in 6-well plates at a density of $2 \times$ $10^{5}$ cells per well and exposed to different concentrations of either PEBP or NBJ. When the cells were $70 \%$ to $80 \%$ confluent, a $1,000 \mu \mathrm{L}$ pipette tip was used to create a scratch in the monolayer. Photos were taken at several different places along the wound at 0 hour, 24 hours and 48 hours. The cell motility assay was analyzed with TScratch software (CSE Lab, Zurich, Switzerland).

\section{Quantitative real-time PCR (qPCR)}

Total RNA was extracted, from cancer cells or CSCs, for both cell lines after 24 hours of exposure to different concentrations of PEBP or NBJ with the miRNeasy Mini Kit (no. 217004; Qiagen, Toronto, ON, Canada) by following the manufacturer's protocol. RNA concentrations were determined with a NanoDrop ND-2000 (Thermo Fisher Scientific, Waltham, MA, USA). RNA extracted in this manner is suitable for measurements of miRNA expression levels.

qPCR was used to measure miRNA and gene expression levels. RNA was reverse-transcribed into cDNA by Moloney murine leukemia virus reverse transcriptase (no. 28025013; Invitrogen) and by using miRNA specific primers purchased from Ambion (Life Technologies, Burlington, ON, Canada). Subsequent miRNA-200b-3p (ID. 002251) levels were quantified by qPCR using Taqman probes (Applied Biosystems, Burlington, ON, Canada) and a FastStart Taq Polymerase
(Roche, Mississauga, ON, Canada), according to manufacturer's protocols. miRNA PCR reactions were conducted at $95^{\circ} \mathrm{C}$ for 10 minutes, followed by 40 cycles of $95^{\circ} \mathrm{C}$ for 30 seconds and $60^{\circ} \mathrm{C}$ for 1 minute. We used snRNA U6 as control. Expression of the indicated miRNAs was measured by comparing cycle threshold value using BIO-RAD CFX96 Manager software. The relative level was calculated using the $\triangle \triangle \mathrm{CT}$ method.

\section{miRNA transfection}

$2 \times 10^{5}$ B16F10 cells could grow to $30 \%$ confluence in DMEM supplemented with FBS and antibiotics (penicillin/streptomycin). Cells were transfected with a miR-200b mimic (ID. MC10492), the Mirvana miR-200b inhibitor (ID. MH10492) or a non-coding control (Ambion; ThermoFisher Scientific) by using lipofectamine 2000 (no. 11668019; Life Technologies) for 24 hours. After incubation, a passage was completed, and cells were plated in regular 6-well or 6 ultra-low attachment plates. After transfection, the expression of miR-200b was measured by qPCR.

\section{Protein extraction}

B16F10 cells were transfected as mentioned above and collected. After washing with PBS on ice, cells were mixed with $400 \mu$ L Pierce ${ }^{\circledR}$ RIPA buffer (no. PI89900; ThermoFisher Scientific) combined with the Halt ${ }^{\mathrm{TM}}$ Protease and Phosphatase Inhibitor Cocktail (1×) (no. RH238230A; ThermoFisher Scientific). Cells were then scraped off the plate and protein mixes were centrifuged to remove the debris.

\section{Western blot analysis}

The protein extract concentration was measured using the Pierce BCA Protein Assay Kit (no. 23227; ThermoFisher Scientific) following the manufacturer's protocol. After normalization, protein lysates were diluted in Laemmli buffer. Ten $\mu \mathrm{g}$ of protein samples were loaded in each well in a Mini Gel Tank (Life Technologies) in MES Running buffer (20x) (no. 1675920; Life Technologies) and migrated at 200 volts for 22 minutes and then transferred to an Immobilon-p50 polyvinylidene difluoride membrane in a Trans-Blot Cell (Bio-Rad, Hercules, CA, USA) cooled by a Neslab machine. Membranes were washed with TBS containing Tween 20 (TBST) and then blocked at room temperature for 1 hour in $5 \%$ milk in TBST. Membranes were incubated with primary antibody for ZEB1 (Anti-AREB6) at a dilution of 1:5,000 (Ab125512; Abcam, Cambridge, United Kingdom) in $5 \%$ bovine serum albumin (no. 23227; Pierce Biotechnology, ThermoFisher Scientific) in TBST, according to manufacturer's recommendations, and incubated with agitation at $4^{\circ} \mathrm{C}$ overnight. The membranes were washed five times for 15 minutes with TBST and then incubated with horseradish peroxidase conjugated anti-rabbit secondary antibody at a final dilution of 1:10,000 (Ab39368; Jackson Immuno Research Laboratories, West Grove, PA, USA) for 1 hour at room temperature. 
The antibodies were detected using PRIME-ECL solutions (no. 9713237; Pierce Biotechnology). The membranes were archived with a VersaDoc (Bio-Rad) and normalized by using $\beta$-actin (Ab8227; Abcam) or $\alpha$-tubulin (Ab4074; Abcam) as a loading control. The densitometry of Western blot results was performed using Image lab software (Bio-Rad).

\section{Statistical analysis}

All experiments were repeated in triplicate. All values are displayed as mean \pm SE (SEM). Statistical significance was determined by one-way ANOVA, post-hoc Tukey test or TwoWay ANOVA on GraphPad Prism 5.0 (GraphPad Software, La Jolla, CA, USA). Biorad Image Lab was using to analyze the Western blot result. Flow cytometry results were analyzed with Kaluza 1.3 software (Beckman Coulter Inc., Montréal, QC, Canada). The cell motility assay was analyzed with TScratch software (CSE Lab, Zurich, Switzerland). $P$-values $<0.05$ were considered as statistically significant.

\section{RESULTS}

\section{PEBP reduces the number and the size of spheres}

The HS 294T and B16F10 malignant melanoma cells were plated in stem cell conditioned culture medium in 6-well plates at a density of 100,000 cells/well. Sphere growth
A

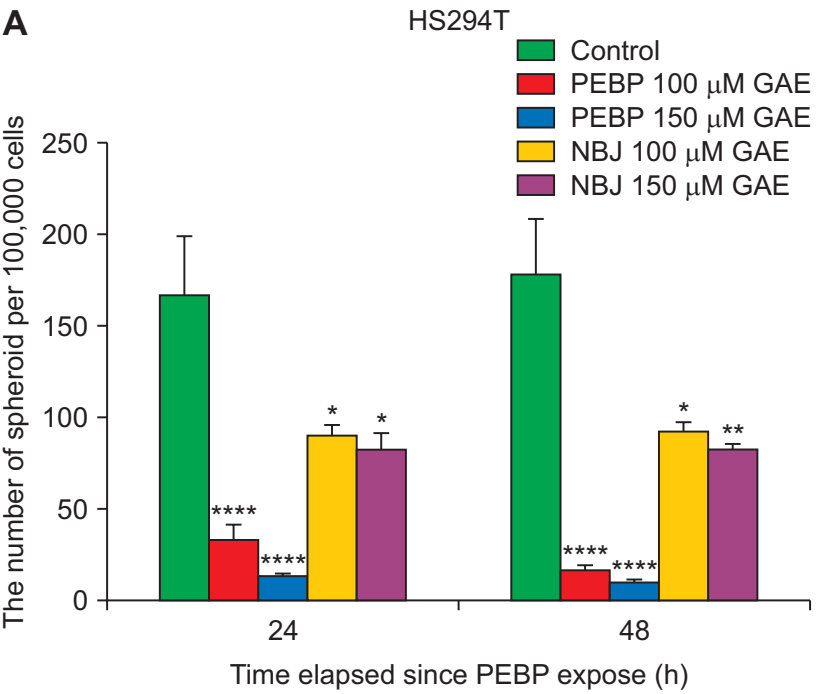

B B16F10

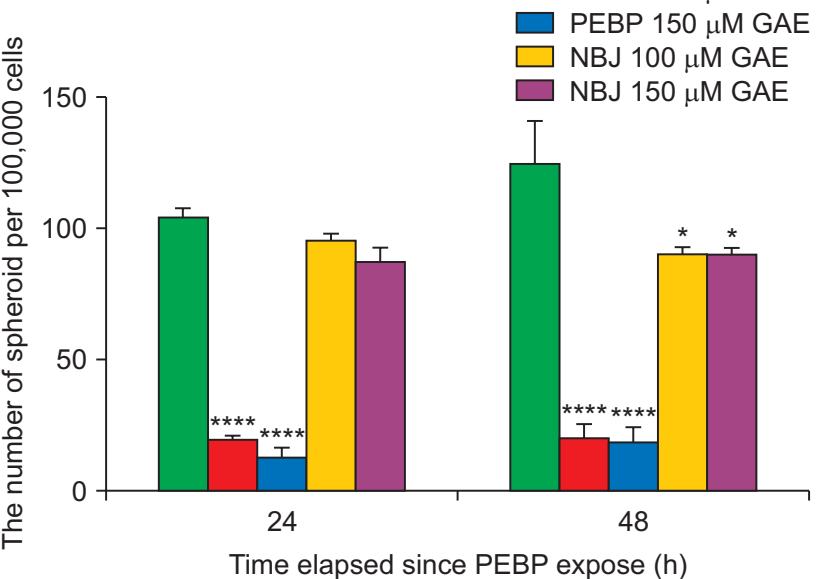

C

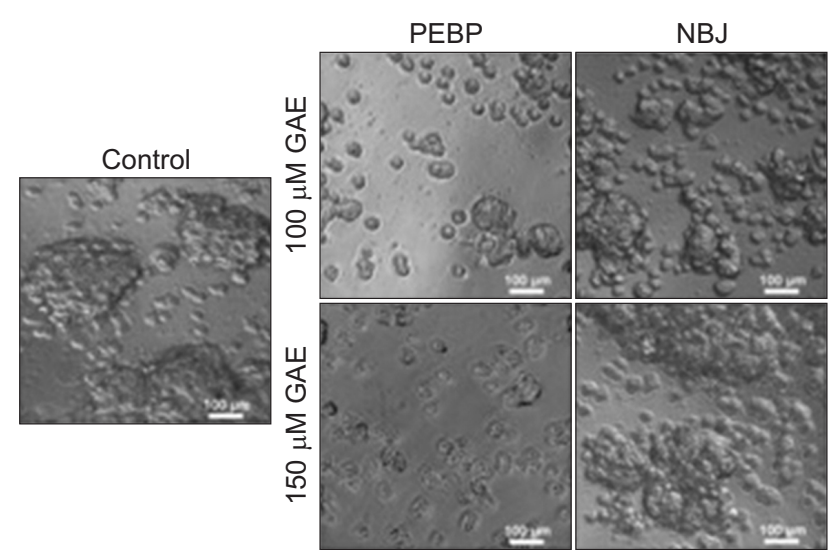

D

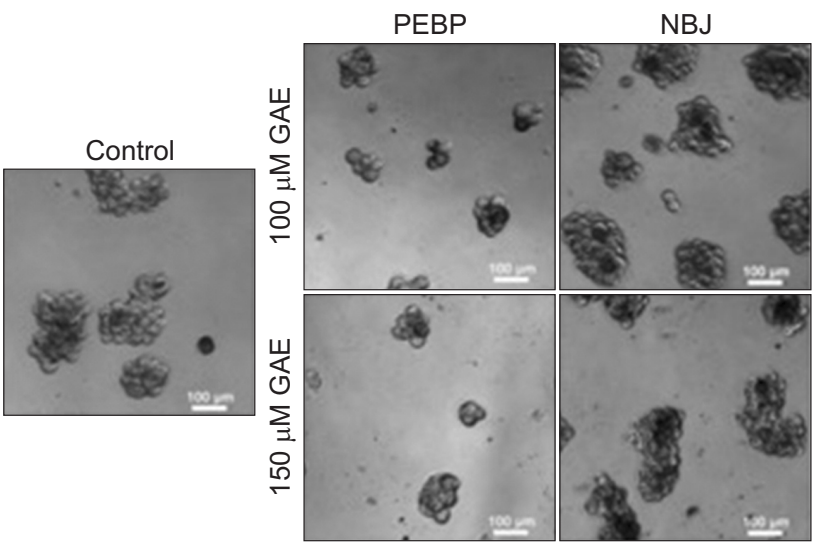

Figure 1. Sphere growth is inhibited after PEBP treatment. Inhibition of human melanoma HS 294T (A) and murine melanoma B16F10 (B) spheroid development after treatment with 100 and $150 \mu \mathrm{M}$ GAE (gallic acid equivalent) of either PEBP or NBJ. Photographs of HS294T (C) and B16F10 (D) spheres taken with AxioCamMR3 camera on light microscope (magnification, $\times 20$ ). After 2 days in culture. Spheres are isolated and grown in 96-well plates at $37^{\circ} \mathrm{C}$ and $5 \% \mathrm{CO}_{2}$. Significance shows as ${ }^{*} P<0.05,{ }^{* *} P<0.01,{ }^{* * * *} P<0.0001$ different from control. Two-Way ANOVA was used. Data represent a combination of 3 experiments. All data are presented as mean \pm SEM. PEBP, polyphenol enriched blueberry preparation; NBJ, non-fermented blueberry juice. 
was measured for 24 hours after exposure to different concentrations of either PEBP or NBJ. PEBP significantly decreased the number of spheroids in both HS 294T (Fig. 1A) and B16F10 (Fig. 1B) cell lines $(P<0.0001)$ and the size of adhered clumps of these cells (Fig. 1C and 1D) growing in non-adherent conditions.

However, the same concentrations of NBJ had much weaker effects on the sphere formation in both cell lines.

\section{PEBP suppresses the expression of CD133 ${ }^{+}$, $\mathrm{CD}_{4}{ }^{+}$, and $\mathrm{CD} 24^{+}$in B16F10 and CD133 ${ }^{+}$and $\mathrm{CD}^{+}{ }^{+}$in HS 294T melanoma skin cancer cells}

To validate the effects of PEBP in the two cell lines, stemess markers were analyzed by flow cytometry. Flow cytometry analysis was used to determine the presence of the cell-surface stem cell markers CD133, CD44, and CD24 in B16F10, and CD133 and CD20 in HS 294T cell lines (Fig. 2). The analysis was carried out following 24 hour treatment with 100 $\mu \mathrm{M}$ GAE of PEBP or NBJ. The result indicated that PEBP reduced the surface marker expression CD133 (1.95\% com- pared to control 5.63\%), CD44 (55.10\%, compared to control $84.17 \%)$ and CD24 (13.39\%, compared to control $17.67 \%)$ on B16F10 cells (Fig. 2A), and reduced the surface marker expression CD20 (13.73\% compared to control $21.80 \%$ ) and CD133 (0.08\% compared to control $2.74 \%$ ) on HS 294T cells (Fig. 2B). However, NBJ treatment caused no significant changes in surface marker expression CD133 (4.84\% compared to control 5.63\%), CD44 (69.25\%, compared to control $84.17 \%)$ and CD24 (21.46\%, compared to control $17.67 \%)$ on B16F10 cells and CD20 (22.30\% compared to control $21.80 \%$ ) and CD133 (2.00\% compared to control $2.74 \%$ ) on HS 294T cells.

\section{PEBP inhibits migration of melanoma cells}

A motility assay was performed to examine the effect of PEBP on migrative capability of HS 294T (Fig. 3A) and B16F10 (Fig. 3B) cells. For this purpose, cells were treated with $100 \mu \mathrm{M}$ GAE and $150 \mu \mathrm{M}$ GAE of PEBP or NBJ for 0 hour, 24 hours and 48 hours. Next, treating cells with PEBP prevented the surface area from being closed compared to
$\mathbf{A}$

FITC-CD133 area B16F10
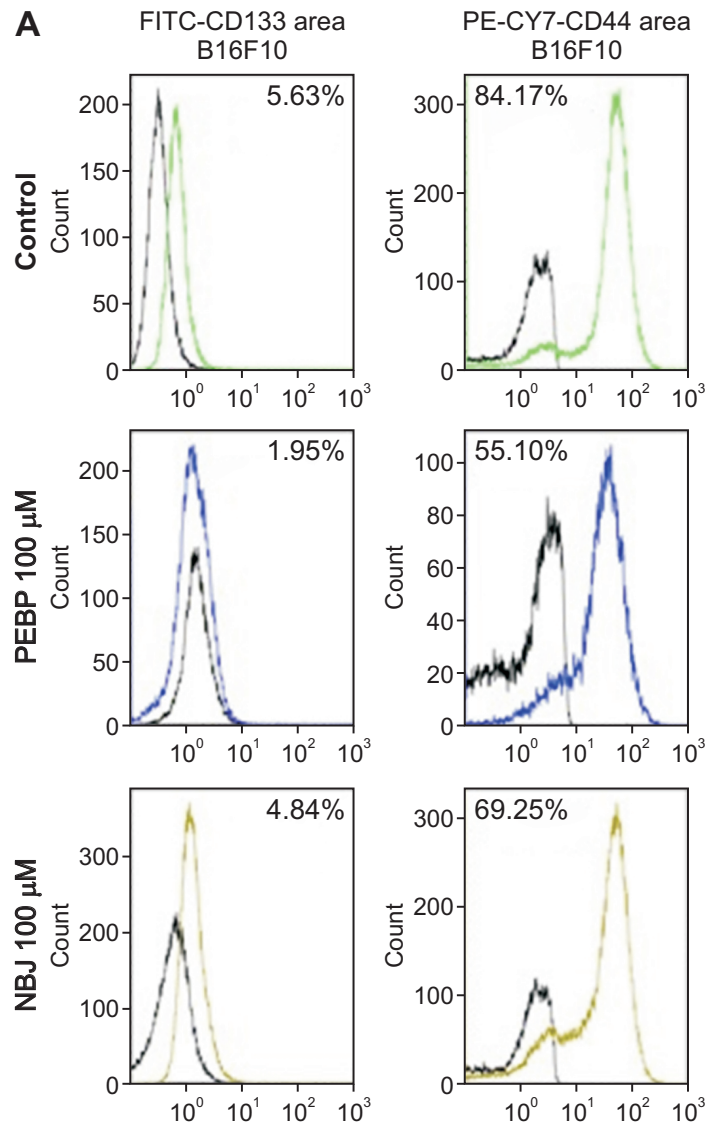
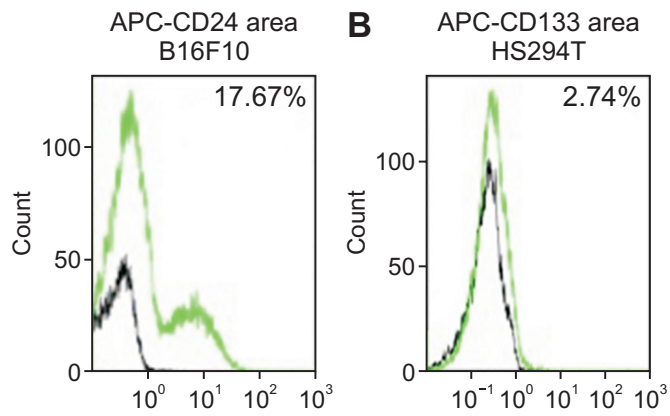

Alexa Fluor 450-CD20 area
HS294T
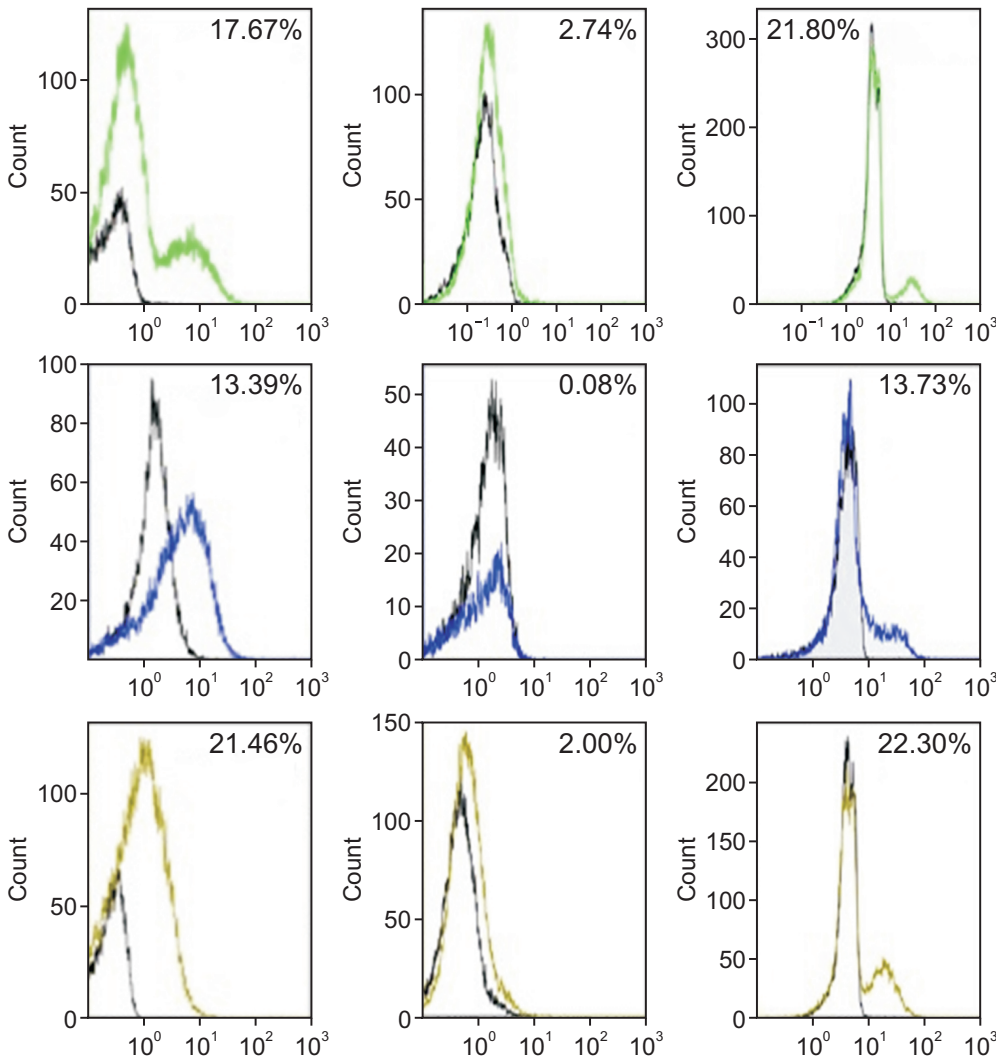

Figure 2. PEBP suppresses the $\mathrm{CD} 133^{+} \mathrm{CD} 44^{+} \mathrm{CD} 24^{+}$phenotype in $\mathrm{B} 16 \mathrm{~F} 10$ and the $\mathrm{CD} 133^{+} \mathrm{CD} 20^{+}$phenotype in HS294T melanoma skin cancer. Effect of 24 hours of exposure to PEBP and NBJ on surface marker expression of cancer stem cells (CSCs) in B16F10 and HS 294T cell lines characterized by flow cytometry. (A) B16F10 CSCs were treated with PEBP (100 $\mu \mathrm{M}$ GAE) and NBJ (100 $\mu \mathrm{M}$ GAE). Suspension cells were labeled with FITC-conjugated anti-CD133, PE-CY7 conjugated anti-CD44 and APC-conjugated anti-CD24 antibodies for B16F12 cells. (B) APCconjugated anti-CD133, Alexa Fluor 450-conjugated anti CD20 for HS 294T cells and analyzed by control flow cytometry. PEBP, polyphenol enriched blueberry preparation; NBJ, non-fermented blueberry juice. 
A

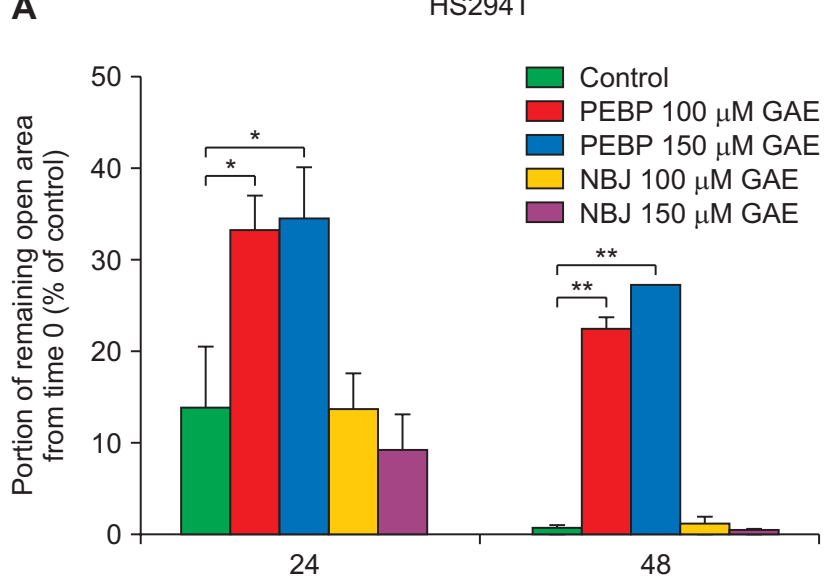

Time elapsed since PEBP expose $(\mathrm{h})$

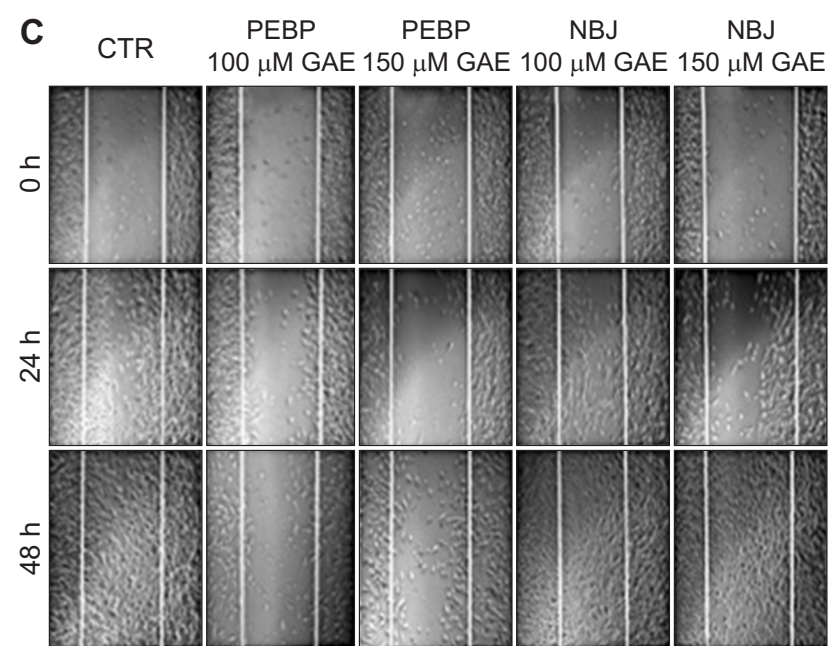

D

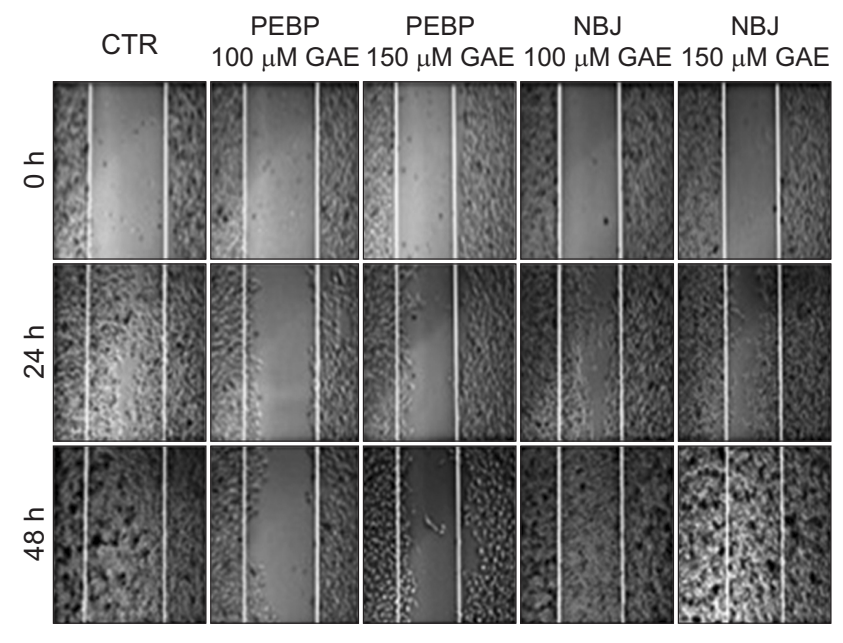

Figure 3. PEBP inhibits migration of melanoma cells. Effects of PEBP on cell motility. (A, B) HS 294T and B16F10 cells exposed to different concentrations of either PEBP or NBJ at 0, 24, and 48 hours. Cells were plated in 6-well plates in Dulbecco's Modified Eagle's Medium (DMEM) and incubated at $5 \% \mathrm{CO}_{2}$ and $37^{\circ} \mathrm{C}$. (C, D) Photographs were taken at indicated time points after scratch injury. Two-Way ANOVA was used to analyze the statistical difference between the groups. Data represent a combination of 3 experiments. All data are presented as mean \pm SEM of relative wound closure. Significance shows as ${ }^{\star} P<0.05,{ }^{* \star} P<0.01,{ }^{* \star *} P<0.001$ different from control. PEBP, polyphenol enriched blueberry preparation; NBJ, nonfermented blueberry juice; CTR, control.

control in both cell lines (Fig. 3C and 3D).

\section{PEBP increases the expression of miR-200b in B16F10 skin cancer cells}

Members of miRNA-200 family are known to be tumor suppressors, which are down-regulated in some types of cancer [28]. To determine the expression level of miR-200b in the metastatic melanoma cell line B16F10, we performed qPCR. We demonstrated that PEBP significantly increased miR$200 \mathrm{~b}$ expression in the B16F10 cell line by approximately 4.3fold in comparison to the control $(P<0.0001)$, whereas NBJ did not have a significant effect on miR-200b expression as shown in Figure 4A. Additionally, we showed that PEBP significantly increased miR-200b expression in B16F10 spher- oids by approximately 3-fold $(P<0.001)$ (Fig. 4B).

\section{PEBP affects the expression of miR-200b in B16F10 melanoma cells transfected with miR- 200b mimic}

Transfection studies were performed to further investigate the role of miR-200b in the malignant murine melanoma cell line. B16F10 cells transfected with a miR-200b mimic and exposed to $100 \mu \mathrm{M}$ GAE of PEBP for 24 hours showed significantly higher expression of $\mathrm{miR} 200 \mathrm{~b}$ than did the control cells $(P<0.0001)$ (Fig. 5). In the skin CSCs, the mimic significantly inhibited the formation of spheres concomitantly with PEBP at a concentration of $100 \mu \mathrm{M}$ GAE (Fig. 6). These results indicate that $\mathrm{PEBP}$ prevents spheroid growth and is 

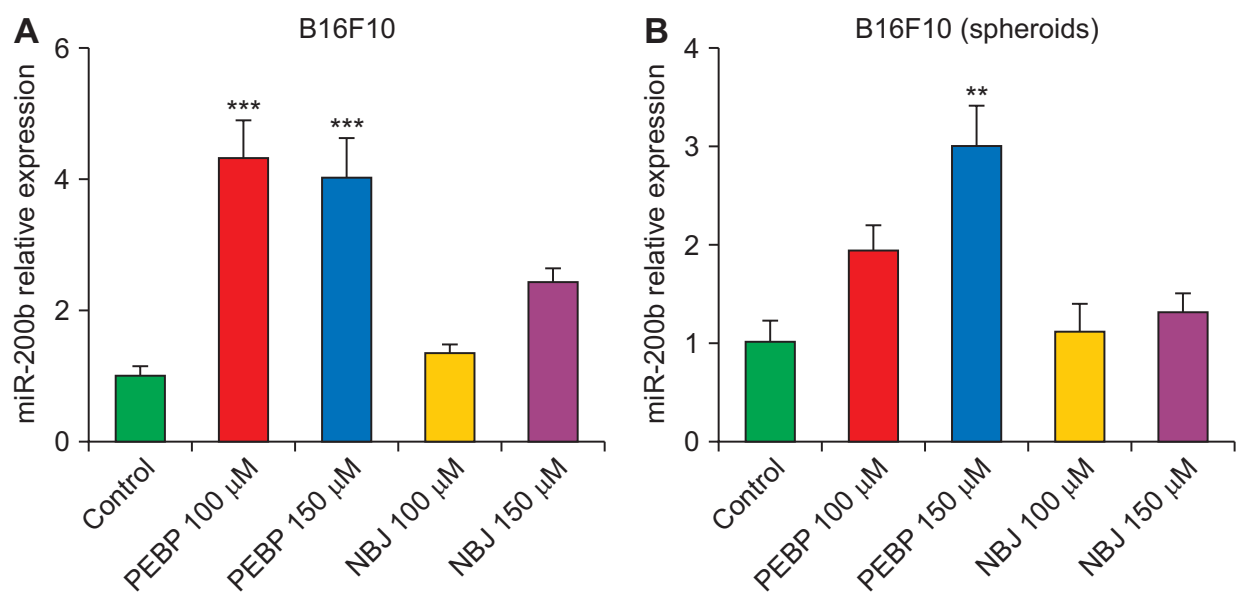

Figure 4. Expression of miR-200b is upregulated in skin cancer. Relative normalized expression (RT2-quantitative real-time PCR [qPCR] analysis) of miR-200b in B16F10 cells after 24 hours exposure to different concentrations of PEBP or NBJ. Cells were plated in (A) 6-well attachment plates in Dulbecco's Modified Eagle's Medium (DMEM) and (B) 6-well ultra-low attachment plates in DMEM-F12 (spheroid medium) and incubated at $5 \% \mathrm{CO}_{2}$ and $37^{\circ} \mathrm{C}$. Data were normalized to the RNA control U6snRNA. One-way ANOVA, followed by post-hoc Tukey's multiple comparisons were used. Data represent a combination of 3 experiments. All data are presented as mean \pm SEM. Significance shows as ${ }^{* \star} P<0.01$, ${ }^{\star \star *} P<0.001$ different from control. PEBP, polyphenol enriched blueberry preparation; NBJ, non-fermented blueberry juice.

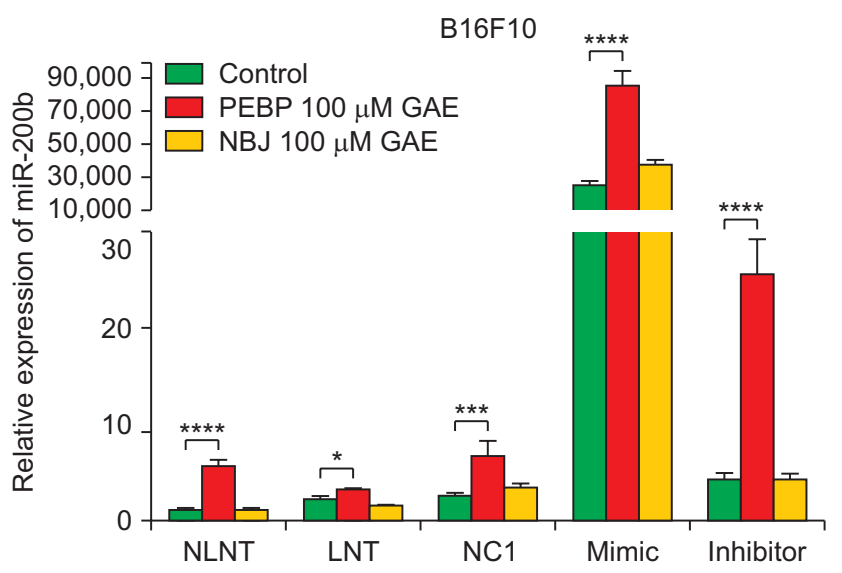

Figure 5. PEBP elevates the expression of miR-200b in transfected B16F10 cells. Relative normalized expression (RT2-quantitative realtime PCR [qPCR] analysis) of miR-200b after 24-hour exposure to either PEBP or NBJ. B16F10 cells could grow to $30 \%$ confluence in DMEM medium. Cells were transfected with miR-200b mimic, antimiR miRNA Inhibitor, control (NLNT), Lipofectamine no target (LNT) and noncoding RNA (NC1) using Lipofectamine 2000 (Invitrogen, Burlington, ON, Canada), and the expression of miR-200b was detected by RT2-qPCR. Two-Way ANOVA was used to analyze the statistical difference between the groups. Data represent a combination of 3 experiments. All data are presented as mean \pm SEM. Significance shows as ${ }^{\star} P<0.05,{ }^{* \star *} P<0.001,{ }^{* * \star *} P<0.0001$ different from control. PEBP, polyphenol enriched blueberry preparation; NBJ, non-fermented blueberry juice.

more effective than NBJ.

\section{PEBP down-regulates ZEB1 expression in B16F10 skin cancer cells}

The effect of miR 200b overexpression on ZEB1 protein expression was determined by Western blot analysis. B16F10 melanoma cells were transfected with the miR-200b mimic or miR-200b inhibitor. A down-regulation of ZEB1 protein expression was observed in cells transfected with the miR$200 \mathrm{~b}$, whereas cells transfected with the miR-200b inhibitor had an increased expression of ZEB1 when compared with control cells $(P<0.05)$ (Fig. 7).

\section{PEBP down-regulates the expression of ZEB1 protein in B16F10 skin cancer cells}

Next, we determined whether PEBP affects EMT in malignant melanoma B16F10 murine melanoma cells. For this purpose, B16F10 cells were treated with PEBP for 48 hours, and cell lysates were prepared for the Western blot analysis of ZEB1. The data show that PEBP decreases the levels of ZEB1 in B16F10 cells compared to untreated controls (Fig. 8). The expression of $\alpha$-tubulin was measured as a reference.

\section{DISCUSSION}

Malignant melanoma is one of the most aggressive and life-threatening cancers originating from melanocytes. Numerous efforts have been made to improve the treatment of malignant melanoma, but no effective therapy is currently available [29]. Chemopreventive strategies are becoming important in translational medicine in oncology [30]. Skin photoprotection is an important aspect of skin cancer prevention against photo-induced damage, the leading cause of skin cancer.

Bio-transformed blueberry juice was recently found to exhibit a variety of anticancer effects, such as protection against DNA damage [31], regulation of important oncogenic cellular signaling pathways [32], inhibition of cancer cell proliferation, induction of apoptosis, modulation of oncogene or tumor sup- 
A

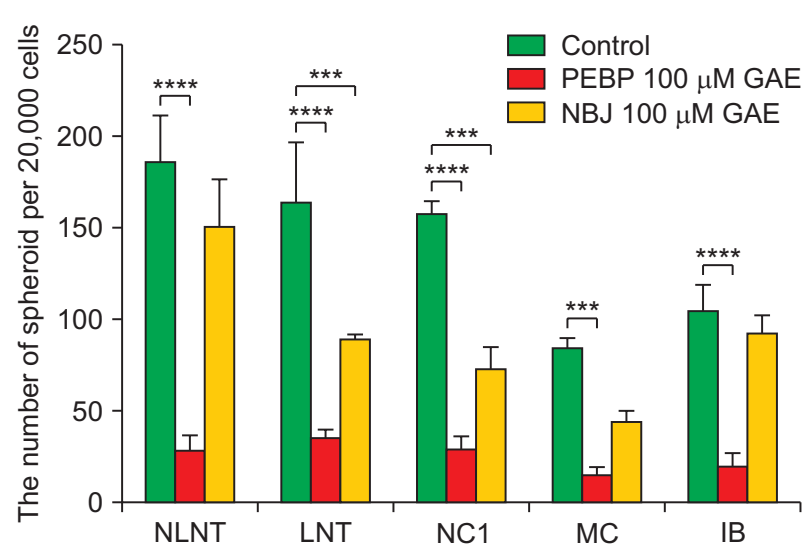

B

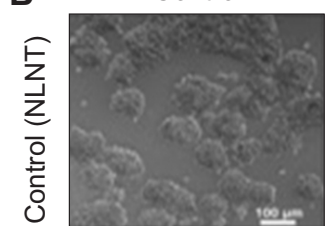

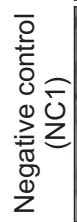
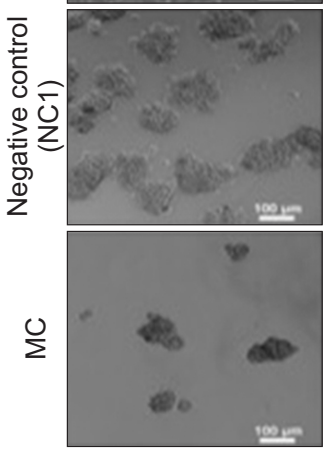

$\underline{\underline{\underline{O}}}$

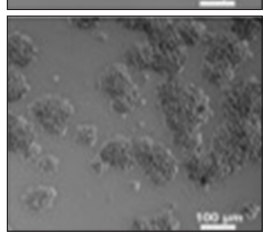

PEBP
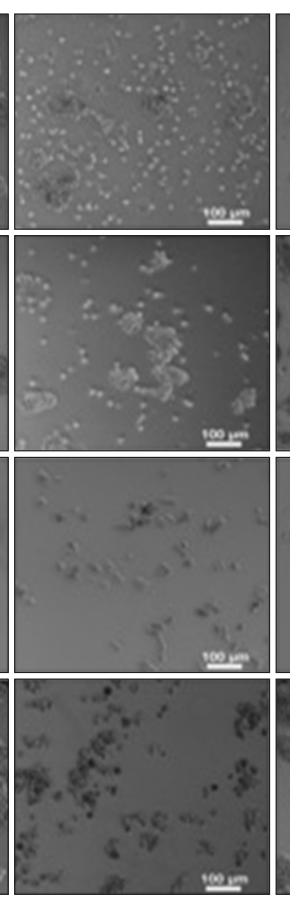

NBJ

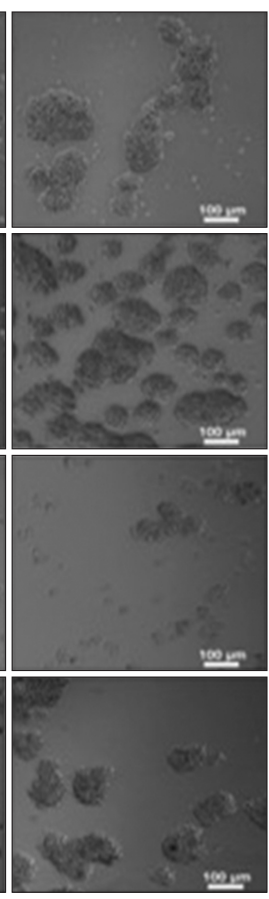

Figure 6. PEBP reduces sphere growth in transfected B16F10 cells. Inhibition of spheroid development derived from the murine melanoma cell line B16F10 after 24-hour exposure to PEBP. (A) B16F10 cells were transfected with miR-200b mimic (MC), anti-miR miRNA Inhibitor (IB), control (NLNT), Lipofectamine no target (LNT) and noncoding RNA (NC1) in 6-ultra-low attachment plates in BMEM-F12 and spheroid medium and incubated at $5 \% \mathrm{CO}_{2}$ and $37^{\circ} \mathrm{C}$. (B) The phenotype of control, PEBP-treated and NBJ-treated cells after 2 days in culture (magnification, $\times 20$ ). TwoWay ANOVA was used to analyze the statistical difference between the groups. Data represent a combination of 3 experiments. All data are presented as mean \pm SEM. Significance shows as ${ }^{\star \star *} P<0.001,{ }^{* \star \star *} P<0.0001$ different from control. PEBP, polyphenol enriched blueberry preparation; NBJ, non-fermented blueberry juice.

pressor gene expression [33]. We have provided evidence that PEBP potently reduces tumor growth and metastasis [5]. In line with these reports, our preliminary results have shown that repression of CSCs in breast cancer cells by fermented blueberry supports a diet-mediated targeting of CSCs by controlling the inflammatory circuits, such STAT3, AKT, PI3K, and ERK1/2 [5], pathways that are involved in the maintenance and development of CSCs from epithelial cancers that also include skin cancer [34]. Therefore, we aimed to study the underlining mechanisms that drive the anticarcinogenic effects of PEBP in malignant melanoma skin cancer and its relationship to the CSC.

It has been known since the 1980s that certain melanoma cell lines have the ability to form spheroids in vitro [35]. The important characteristic of melanoma spheroids is that they exhibit more chemoresistance than when grown in monolayers $[36,37]$. This feature is considered to further reflect stem cell-like behavior [38]. Controlling CSC growth in skin cancer is a possible avenue to prevent tumor development and metastasis. Thus, the investigation of PEBP-induced molecular mechanisms that mediate CSC growth was important to clarify its anticancer and anti-metastatic activities. Our data indicated that PEBP significantly inhibited spheroid growth in B16F10 and HS 294T cells, suggesting that its effect is not cell type dependent. The mechanism that occurs during fermentation might explain why PEBP showed better inhibitory effects on CSCs compared to the unfermented control and normal blueberry juice, as shown in Figure 1. PEBP has antioxidant potential that endows it with novel anti-inflammatory [39], antidiabetic [40,41] and neuroprotective [6] biological properties. During fermentation, long chain polyphenols are subject to hydrolysis by microbial enzymes, which render them more bioavailable and more bio-functional, thus facilitating their transdermal absorption, and potentially increasing their photoprotective and anti-oxidant capacities [42].

Cell proliferation and motility are two characteristics required for tumor progression. The expansion of a primary tumor mass is related to an increase in proliferation [43]. The cell motility is important in order to disseminate a primary tumor from one site to another, leading to an invasive phenotype [44]. In the present study, cell proliferation and the migration were investigated in metastatic B16F10 and HS 294T melanoma cells. These data suggest that PEBP significantly inhibited the motility of both cancer cell lines compared to the control cell line and was more potent than NBJ. Therefore, PEBP could have an effective role in the management of melanoma.

Melanoma cell lines express stem cell-associated surface 
A
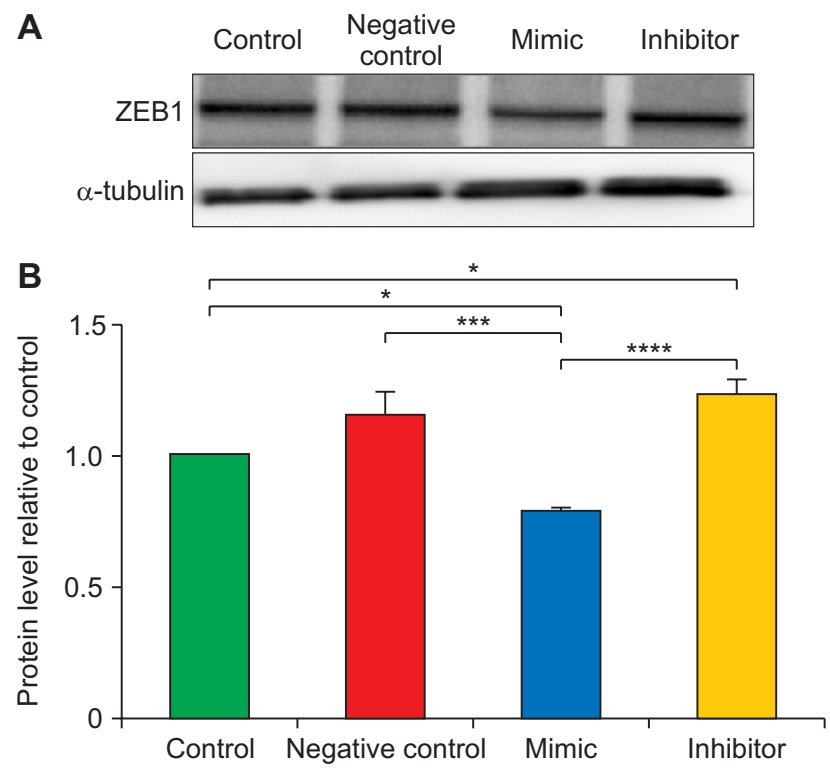

Figure 7. miR-200b inhibits ZEB1 expression in transfected B16F10 cells. (A) Sample Western blot photographs for ZEB1 and control $\beta$-actin as shown on Image Lab. (B) Combined Western blot data from Image Lab calculations. B16F10 melanoma cells were transfected with a negative control, mimic, or inhibitor and analyzed by Western blot. One-way ANOVA, followed by post-hoc Tukey's multiple comparisons was used. Data represent a combination of 3 experiments. All data are presented as mean \pm SEM. Significance shows as ${ }^{*} P<0.05,{ }^{* * *} P<0.001,{ }^{* * * *} P<0.0001$ different from control. ZEB1, zinc-finger and E-box binding homeobox 1 .

markers. Some of the CSC surface markers have been identified, including CD133, CD44, and CD24 in B16F10 cells [13]. CD133 and CD20 were identified in HS 294T cells [45]. PEBP induced a significantly lower expression of CD133 in B16F10 and HS294T cell lines in comparison to the NBJ-treated cells and control groups. CD133 regulates metastatic disease and cell growth, and it has been described as a marker of malignant melanoma skin CSCs [46,47]. Further, CSC potential of self-renewal and differentiation was shown to be exclusively contained within tumor cell subsets characterized by the expression of the CD133 stem cell marker [48]. These markers could be valuable tools towards the development of new strategies of treatment and chemoprevention of skin cancer by natural compounds.

Several miRNAs associated with different clinical-pathological characteristics of tumors such as stemness, invasion and chemoresistance are involved in sustaining an inflammatory microenvironment that favors neoplasia and CSCs. In addition, some studies have reported a correlation between miRNA expression and development of tumor [49]. Particularly, many research groups have shown that miR-200b functionally acts as a tumor suppressor by inhibiting cancer cell proliferation and migration by inhibiting the EMT process in a wide range of human malignances [28,50-52]. It has been identified that miR-200b is greatly reduced in melanomas $[26,53,54]$.
A
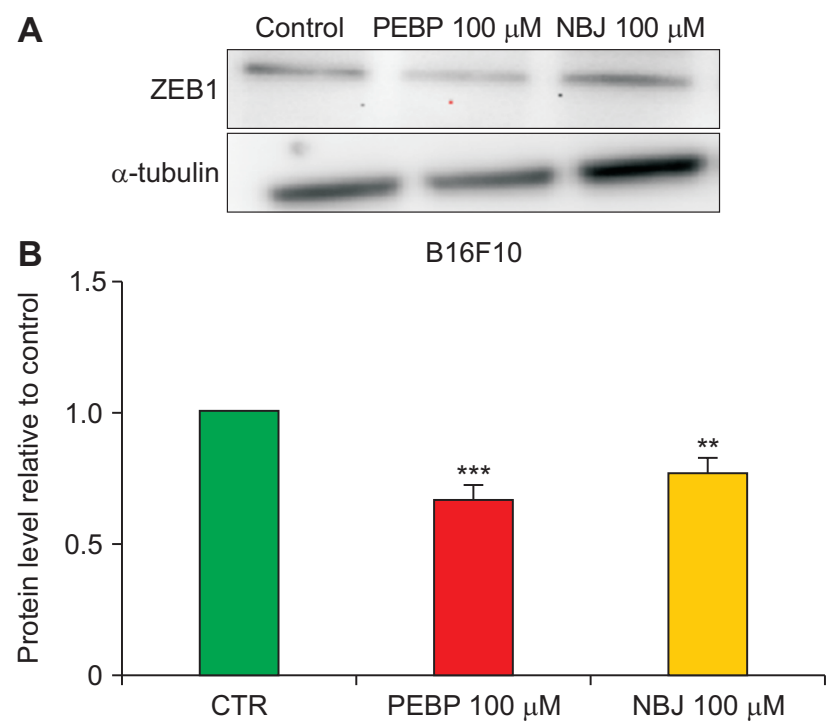

Figure 8. PEBP down-regulates ZEB1 expression in B16F10 cells. (A) Sample Western blot photographs for ZEB1 and control $\alpha$-tubulin as shown on Image Lab. (B) Combined Western blot data from Image Lab calculations. Samples were treated by either polyphenol enriched blueberry preparation (PEBP) or non-fermented blueberry juice (NBJ) for 48 hours and detected by Western blot. One-way ANOVA, followed by post-hoc Tukey's multiple comparisons was used. Data represent a combination of 5 experiments. All data are presented as mean \pm SEM. Significance shows as ${ }^{* *} P<0.01,{ }^{* \star *} P<0.001$ different from control. ZEB1, zinc-finger and E-box binding homeobox 1; PEBP, polyphenol enriched blueberry preparation; NBJ, non-fermented blueberry juice; CTR, calcitonin receptor.

miR-200b was further investigated in this project for its potential role in migration and proliferation of melanoma and CSCs. A metastatic melanoma cell line model was used to overexpress miR-200b. In our study, we investigated the role of PEBP on tumor suppressor miR-200b, which is known to reduce metastasis and tumor proliferation [55]. We found that miR-200b was significantly upregulated ( $\sim .3$ fold) by PEBP compared to the control. This result suggests that PEBP influences the expression of miR-200b by upregulating miR$200 \mathrm{~b}$. As melanoma progresses, miR-200b levels decrease. Therefore, the overexpression of miR-200b can revert the melanoma models to have a less aggressive phenotype (Fig. 4A). miR-200b showed a modest increase in cells transfected with a mimic (Fig. 4B). This could be due to the relatively high endogenous expression of miR-200b. Our results indicate that miR-200b expression upregulated by PEBP might provide useful information in the evaluation prognosis for melanoma patients.

An important relationship between miR-200b, ZEB1 and E-cadherin is well established in the literature $[24,51,52,56]$. miR-200b suppresses metastasis and migration by targeting some protein pathways such as ZEB1/2 in a variety of different cellular contexts. Therefore, we analyzed protein from the transfected B16F10 cells. Our results showed an inverse relationship between the expression of miR-200b and ZEB1 in 
B16F10. The expression of ZEB1 was significantly down regulated by over expression miR-200b (Fig. 8). Since miR-200b reduced the expression of ZEB1, we speculated that this might enhance E-cadherin expression in B16F10, resulting in tumor malignancy suppression [57]. These data collectively suggest that miR-200b regulates the EMT in skin cancer cells and CSCs, and this might be a novel way of reversing tumor progression.

In conclusion, in the present study, we found that treatment of B16F10 cells with PEBP resulted in the suppression or loss of ZEB1 (Fig. 8), which suggests that PEBP has the ability to reverse the EMT process in B16F10 cells. This may be one of the possible mechanisms through which PEBP reduce the invasiveness of B16F10 cells, thereby inhibiting their invasion, which help to reduce the metastasis. This study demonstrated that PEBP has a strong relationship with miR-200b pathways in different biological settings and that it could possibly target ZEB1 protein through miR-200b in B16F10 cell line. To sum up all our data, PEBP was shown to effectivelly target CSC which making it an interesting natural product for further studies.

\section{ACKNOWLEDGMENTS}

This research project is financially supported by the Canadian Institutes of Health Research (CIHR) and by a full academic scholarship from the government of Saudi Arabia.

\section{CONFLICTS OF INTEREST}

No potential conflicts of interest were disclosed.

\section{ORCID}

Nawal Alsadi, https://orcid.org/0000-0001-7752-6658

Jean-François Mallet, https://orcid.org/0000-0003-1612-9063

Chantal Matar, https://orcid.org/0000-0003-0346-4751

\section{REFERENCES}

1. Chinery R, Beauchamp RD, Shyr Y, Kirkland SC, Coffey RJ, Morrow JD. Antioxidants reduce cyclooxygenase-2 expression, prostaglandin production, and proliferation in colorectal cancer cells. Cancer Res 1998;58:2323-7.

2. Afaq F, Katiyar SK. Polyphenols: skin photoprotection and inhibition of photocarcinogenesis. Mini Rev Med Chem 2011;11:1200-15.

3. Boniol M, Autier P, Boyle P, Gandini S. Cutaneous melanoma attributable to sunbed use: systematic review and meta-analysis. BMJ 2012;345:e4757.

4. Seema P. Blueberry as functional food and dietary supplement: the natural way to ensure holistic health. Mediterr J Nutr Metab 2014;7:133-43.

5. Vuong T, Mallet JF, Ouzounova M, Rahbar S, Hernandez-Vargas
$\mathrm{H}$, Herceg $\mathrm{Z}$, et al. Role of a polyphenol-enriched preparation on chemoprevention of mammary carcinoma through cancer stem cells and inflammatory pathways modulation. J Transl Med 2016;14:13.

6. Vuong T, Matar C, Ramassamy C, Haddad PS. Biotransformed blueberry juice protects neurons from hydrogen peroxideinduced oxidative stress and mitogen-activated protein kinase pathway alterations. Br J Nutr 2010;104:656-63.

7. Adebo OA, Gabriela Medina-Meza I. Impact of fermentation on the phenolic compounds and antioxidant activity of whole cereal grains: a mini review. Molecules 2020;25:927.

8. Nguyen LV, Vanner R, Dirks P, Eaves CJ. Cancer stem cells: an evolving concept. Nat Rev Cancer 2012;12:133-43.

9. Conley SJ, Wicha MS. Breast cancer stem cells: from theory to therapy. In: Sell S, ed. Stem Cells Handbook. New York, Springer, pp 477-89, 2013.

10. Allan AL, Vantyghem SA, Tuck AB, Chambers AF. Tumor dormancy and cancer stem cells: implications for the biology and treatment of breast cancer metastasis. Breast Dis 20062007;26:87-98.

11. Kumar R, Sharma A, Pattnaik AK, Varadwaj PK. Stem cells: an overview with respect to cardiovascular and renal disease. J Nat Sci Biol Med 2010;1:43-52.

12. Tirino V, Desiderio V, Paino F, De Rosa A, Papaccio F, La Noce $M$, et al. Cancer stem cells in solid tumors: an overview and new approaches for their isolation and characterization. FASEB $\mathrm{J}$ 2013;27:13-24.

13. Dou J, Pan M, Wen P, Li Y, Tang Q, Chu L, et al. Isolation and identification of cancer stem-like cells from murine melanoma cell lines. Cell Mol Immunol 2007;4:467-72.

14. Kong D, Li Y, Wang Z, Sarkar FH. Cancer stem cells and Epithelial-to-Mesenchymal Transition (EMT)-phenotypic cells: are they cousins or twins? Cancers (Basel) 2011;3:716-29.

15. Abdullah LN, Chow EK. Mechanisms of chemoresistance in cancer stem cells. Clin TransI Med 2013;2:3.

16. Lehmann W, Mossmann D, Kleemann J, Mock K, Meisinger C, Brummer T, et al. ZEB1 turns into a transcriptional activator by interacting with YAP1 in aggressive cancer types. Nat Commun 2016;7:10498.

17. Liu Y, El-Naggar S, Darling DS, Higashi Y, Dean DC. Zeb1 links epithelial-mesenchymal transition and cellular senescence. Development 2008;135:579-88.

18. Zetter BR. Adhesion molecules in tumor metastasis. Semin Cancer Biol 1993;4:219-29.

19. Richards EJ. Inherited epigenetic variation--revisiting soft inheritance. Nat Rev Genet 2006;7:395-401.

20. Blanpain C, Fuchs E. Epidermal stem cells of the skin. Annu Rev Cell Dev Biol 2006;22:339-73.

21. He L, Hannon GJ. MicroRNAs: small RNAs with a big role in gene regulation. Nat Rev Genet 2004;5:522-31.

22. Bartel DP. MicroRNAs: genomics, biogenesis, mechanism, and function. Cell 2004;116:281-97.

23. Gebeshuber CA, Zatloukal K, Martinez J. miR-29a suppresses tristetraprolin, which is a regulator of epithelial polarity and 
Alsadi et al.

metastasis. EMBO Rep 2009;10:400-5.

24. Park SM, Gaur AB, Lengyel E, Peter ME. The miR-200 family determines the epithelial phenotype of cancer cells by targeting the E-cadherin repressors ZEB1 and ZEB2. Genes Dev 2008;22:894-907.

25. Zhang J, Ma L. MicroRNA control of epithelial-mesenchymal transition and metastasis. Cancer Metastasis Rev 2012;31:65362.

26. Xu Y, Brenn T, Brown ER, Doherty V, Melton DW. Differential expression of microRNAs during melanoma progression: miR200c, miR-205 and miR-211 are downregulated in melanoma and act as tumour suppressors. Br J Cancer 2012;106:553-61.

27. Peng F, Jiang J, Yu Y, Tian R, Guo X, Li X, et al. Direct targeting of SUZ12/ROCK2 by miR-200b/c inhibits cholangiocarcinoma tumourigenesis and metastasis. Br J Cancer 2013;109:3092104.

28. Yao Y, Hu J, Shen Z, Yao R, Liu S, Li Y, et al. MiR-200b expression in breast cancer: a prognostic marker and act on cell proliferation and apoptosis by targeting Sp1. J Cell Mol Med 2015;19:760-9.

29. Ma J, Frank MH. Tumor initiation in human malignant melanoma and potential cancer therapies. Anticancer Agents Med Chem 2010;10:131-6.

30. Pandey KB, Rizvi SI. Plant polyphenols as dietary antioxidants in human health and disease. Oxid Med Cell Longev 2009;2:270-8.

31. Freese R. Markers of oxidative DNA damage in human interventions with fruit and berries. Nutr Cancer 2006;54:143-7.

32. Adams LS, Phung S, Yee N, Seeram NP, Li L, Chen S. Blueberry phytochemicals inhibit growth and metastatic potential of MDA-MB-231 breast cancer cells through modulation of the phosphatidylinositol 3-kinase pathway. Cancer Res 2010;70:3594-605.

33. Seeram NP, Adams LS, Zhang Y, Lee R, Sand D, Scheuller $\mathrm{HS}$, et al. Blackberry, black raspberry, blueberry, cranberry, red raspberry, and strawberry extracts inhibit growth and stimulate apoptosis of human cancer cells in vitro. J Agric Food Chem 2006;54:9329-39.

34. Yang L, Shi P, Zhao G, Xu J, Peng W, Zhang J, et al. Targeting cancer stem cell pathways for cancer therapy. Signal Transduct Target Ther 2020;5:8.

35. Rofstad EK, Wahl A, Davies Cde L, Brustad T. Growth characteristics of human melanoma multicellular spheroids in liquid-overlay culture: comparisons with the parent tumour xenografts. Cell Tissue Kinet 1986;19:205-16.

36. Smalley KS, Haass NK, Brafford PA, Lioni M, Flaherty KT, Herlyn M. Multiple signaling pathways must be targeted to overcome drug resistance in cell lines derived from melanoma metastases. Mol Cancer Ther 2006;5:1136-44.

37. Kalirai H, Damato BE, Coupland SE. Uveal melanoma cell lines contain stem-like cells that self-renew, produce differentiated progeny, and survive chemotherapy. Invest Ophthalmol Vis Sci 2011;52:8458-66.

38. Larson AR, Lee CW, Lezcano C, Zhan Q, Huang J, Fischer AH, et al. Melanoma spheroid formation involves laminin-associated vasculogenic mimicry. Am J Pathol 2014;184:71-8.

39. Vuong T, Martin L, Matar C. Antioxidant activity of fermented berry juices and their effects on nitric oxide and tumor necrosis factor- $\alpha$ production in macrophages 264.7 gamma no(-) cell line. J Food Biochem 2006;30:249-68.

40. Vuong T, Martineau LC, Ramassamy C, Matar C, Haddad PS. Fermented Canadian lowbush blueberry juice stimulates glucose uptake and AMP-activated protein kinase in insulin-sensitive cultured muscle cells and adipocytes. Can J Physiol Pharmacol 2007:85:956-65.

41. Vuong T, Benhaddou-Andaloussi A, Brault A, Harbilas D, Martineau LC, Vallerand D, et al. Antiobesity and antidiabetic effects of biotransformed blueberry juice in $\mathrm{KKA}(\mathrm{y})$ mice. Int $\mathrm{J}$ Obes (Lond) 2009;33:1166-73.

42. Pérez-Gregorio R, Soares S, Mateus N, de Freitas V. Bioactive peptides and dietary polyphenols: two sides of the same coin. Molecules 2020;25:3443.

43. van Diest PJ, Brugal G, Baak JP. Proliferation markers in tumours: interpretation and clinical value. J Clin Pathol 1998;51:716-24.

44. Quaranta V. Motility cues in the tumor microenvironment. Differentiation 2002;70:590-8.

45. Taddei ML, Giannoni E, Morandi A, Ippolito L, Ramazzotti $\mathrm{M}$, Callari $\mathrm{M}$, et al. Mesenchymal to amoeboid transition is associated with stem-like features of melanoma cells. Cell Commun Signal 2014;12:24.

46. Kim M, Koh YJ, Kim KE, Koh BI, Nam DH, Alitalo K, et al. CXCR4 signaling regulates metastasis of chemoresistant melanoma cells by a lymphatic metastatic niche. Cancer Res 2010;70:10411-21.

47. Mimeault M, Batra SK. Novel biomarkers and therapeutic targets for optimizing the therapeutic management of melanomas. World J Clin Oncol 2012;3:32-42.

48. Schatton T, Frank MH. Cancer stem cells and human malignant melanoma. Pigment Cell Melanoma Res 2008;21:39-55.

49. Heinzelmann J, Unrein A, Wickmann U, Baumgart S, Stapf $M$, Szendroi A, et al. MicroRNAs with prognostic potential for metastasis in clear cell renal cell carcinoma: a comparison of primary tumors and distant metastases. Ann Surg Oncol 2014;21:1046-54.

50. Gregory PA, Bert AG, Paterson EL, Barry SC, Tsykin A, Farshid $G$, et al. The miR-200 family and miR-205 regulate epithelial to mesenchymal transition by targeting ZEB1 and SIP1. Nat Cell Biol 2008;10:593-601.

51. Korpal M, Lee ES, Hu G, Kang Y. The miR-200 family inhibits epithelial-mesenchymal transition and cancer cell migration by direct targeting of E-cadherin transcriptional repressors ZEB1 and ZEB2. J Biol Chem 2008;283:14910-4.

52. Burk U, Schubert J, Wellner U, Schmalhofer O, Vincan E, Spaderna S, et al. A reciprocal repression between ZEB1 and members of the miR-200 family promotes EMT and invasion in cancer cells. EMBO Rep 2008;9:582-9.

53. Chen J, Feilotter HE, Paré GC, Zhang X, Pemberton JG, Garady C, et al. MicroRNA-193b represses cell proliferation and 
regulates cyclin D1 in melanoma. Am J Pathol 2010;176:2520-9.

54. Schliekelman MJ, Gibbons DL, Faca VM, Creighton CJ, Rizvi ZH, Zhang Q, et al. Targets of the tumor suppressor miR-200 in regulation of the epithelial-mesenchymal transition in cancer. Cancer Res 2011;71:7670-82.

55. Williams LV, Veliceasa D, Vinokour E, Volpert OV. miR-200b inhibits prostate cancer EMT, growth and metastasis. PLoS One 2013;8:e83991.
56. Christoffersen NR, Silahtaroglu A, Orom UA, Kauppinen S, Lund $A H$. miR-200b mediates post-transcriptional repression of ZFHX1B. RNA 2007;13:1172-8.

57. Wang X, He X, Zhao F, Wang J, Zhang H, Shi F, et al. Regulation gene expression of miR200c and ZEB1 positively enhances effect of tumor vaccine B16F10/GPI-IL-21 on inhibition of melanoma growth and metastasis. J TransI Med 2014;12:68. 